J. Lake Sci. (湖泊科学), 2020, 32(4): 1029-1040

DOI 10. 18307/2020. 0412

(c) 2020 by Journal of Lake Sciences

\title{
鄱阳湖水体溶解有机质分子量分布、荧光特征及对重金属分布的 影响”
}

\author{
李雅妮 ${ }^{1,2}$, , 徐华成 $^{1 * *}$, , 江和龙 ${ }^{1}$ \\ (1:中国科学院南京地理与湖泊研究所湖泊与环境国家重点实验室,南京 210008) \\ (2: 中国科学院大学,北京 100049)
}

\begin{abstract}
摘 要: 溶解有机质 (DOM) 在环境水体中广泛存在, 具有连续的分子量分布特征, 并可显著影响水体中污染物的迁移转 化和归趋行为. 于 2019 年春季采集鄱阳湖全湖水样, 采用超过滤技术将 DOM 样品分为低分子量 (LMW, $<1 \mathrm{kDa}$ ) 和高分 子量 ( HMW , $1 \mathrm{kDa} \sim 0.45 \mu \mathrm{m}$ ) 组分, 通过三维荧光光谱结合平行因子分析 (EEM-PARAFAC) 和 ICP-MS 分析不同分子量区 间有机组分和重金属含量. 结果表明, 鄱阳湖水体 DOM 中 LMW-DOM 占比为 $43 \% \sim 55 \%$, HMW-DOM 占比为 $45 \% \sim 57 \%$. 平行因子模型提取出 3 个相互独立的菼光组分, 分别为类富里酸组分 $\mathrm{C} 1 、 \mathrm{C} 2$ 和类腐殖酸组分 $\mathrm{C} 3.60 \% \sim 73 \%$ 的 C1 分布在 LMW 中, 77\% 93\% 的 C2 分布在 HMW 中, 而 C3 几乎平均分布在 LMW-和 HMW-DOM 中, 可能与 C1、C2、C3 组分分别含 有的传统荧光峰有关. 通过对苂光参数 $F I X(<1.4) 、 B I X(<0.8)$ 的计算发现鄱阳湖 DOM 陆源特征显著, 且 HMW 组分具有 比 LMW 组分更高的陆源特征和芳香度. 重金属检测结果表明 $\mathrm{Cr} 、 \mathrm{Mn} 、 \mathrm{Fe} 、 \mathrm{~Pb}$ 则主要分布在 HMW 中 $(63 \% \sim 94 \%), \mathrm{As}$ 、 $\mathrm{Cd} 、 \mathrm{Ba} 、 \mathrm{Se} 、 \mathrm{Sb}$ 主要分布在 LMW 中 $(63 \% \sim 84 \%), \mathrm{Cu} 、 \mathrm{Zn} 、 \mathrm{Ni} 、 \mathrm{Co}$ 几乎均匀分布在 LMW-和 HMW-DOM 中. 重金属与 DOM 的 相关性结果表明大多数金属与 $\mathrm{C} 2$ 占比呈负相关, 与 $\mathrm{C} 3$ 占比呈正相关, 说明重金属离子与水体中 DOM 的络合可能主要 通过腐殖类组分 $\mathrm{C} 3$ 进行. 且重金属含量的分布受 HMW-DOM 的影响较大, 说明人类活动也对鄱阳湖水体的重金属分布 有着重要影响.
\end{abstract}

关键词: 鄱阳湖;溶解有机质;超过滤;三维苂光; 重金属

Molecular weight distribution, fluorescence characteristics of dissolved organic matter and their effect on the distribution of heavy metals of Lake Poyang*

\author{
LI Yani ${ }^{1,2}$, XU Huacheng ${ }^{1 * *}$ \& JIANG Helong ${ }^{1}$ \\ (1: State Key Laboratory of Lake Science and Environment, Nanjing Institute of Geography and Limnology, Chinese Academy \\ of Sciences, Nanjing 210008, P.R.China) \\ (2: University of Chinese Academy of Sciences, Beijing 100049, P.R.China)
}

Abstract: Dissolved organic matter (DOM) is ubiquitous in environmental waters. It has a characteristic of continuous molecular weight distribution and can significantly affect the migration, transformation, and fate of pollutants in natural waters. Water samples of Lake Poyang were collected in the spring of 2019, and DOM samples were divided into low molecular weight ( LMW, <1 kDa) and high molecular weight ( HMW, $1 \mathrm{kDa}-0.45 \mu \mathrm{m}$ ) components through ultrafiltration. Fluorescence parallel factor (PARAFAC) analysis and ICP-MS were used to analyze the spectral and metal properties in different molecular weight solutions. Results showed that 43\%-55\% of Lake Poyang's DOC was LMW, and 45\%-57\% HMW. DOM fluorescence samples were identified as two fulvic-like components ( C1, C2 ) and a humic-like component C3, of which $60 \%-73 \%$ of $\mathrm{C} 1$ was distributed in LMW, $77 \%-93 \%$ of $\mathrm{C} 2$ distributed in HMW, and C3 almost evenly distributed in the LMW-and HMW-DOM, which may be related to the traditional fluorescent peak contained in each fluorescent components. Calculation of fluorescence index $($ FIX, <1.4) and biological index $(B I X,<0.8)$ indicates that the DOM of Lake Poyang's terrestrial source characteristics were significant, and the

* 2019-09-29 收稿;2019-11-30 收修改稿.

国家自然科学基金项目 (51839011, 51922101)资助.

** 通信作者; E-mail:hcxu@ niglas.ac.cn. 
HMW-DOM had higher terrigenous characteristics and aromaticity than the LMW-DOM. Heavy metal $\mathrm{Cr}, \mathrm{Mn}, \mathrm{Fe}$, and $\mathrm{Pb}$ were mainly distributed in HMW $(63 \%-94 \%)$, As, Cd, Ba, Se, and Sb were mainly distributed in LMW (63\%-84\%), and Cu, $\mathrm{Zn}$, Ni, and Co were almost evenly distributed in LMW- and HMW-DOM. Most metals had negative correlations with C2 percentage and positive correlation with C3 percentage, indicating that the complexation of heavy metal ions with DOM in water may be mainly through humic-like component C3. The distribution of heavy metal content was greatly affected by HMW-DOM, indicating the important role of anthropogenic activities in Lake Poyang's heavy metal distribution.

Keywords: Lake Poyang; dissolved organic matter; ultrafiltration; EEM-PARAFAC; heavy metal

溶解有机质 (dissolved organic matter, DOM) 指水中能通过 $0.45 \mu \mathrm{m}$ 微孔滤膜的组分 ${ }^{[1]}$, 其粒径较小、比 表面积大、分子量分布范围广, 可显著影响水体透明度以及湖泊生态系统的物质和能量循环. 此外, 由于 DOM 包含醌羟基、酮羟基、酚差基、醇羟基和羧基在内的众多活性基团,使其能与水体中众多物质进行离子 交换、表面吸附、络合、鳌合等一系列反应 ${ }^{[2]}$.

环境水体中 DOM 浓度一般 $<20 \mathrm{mg} / \mathrm{L}$, 河水和地下水中一般约为 $2 \sim 10 \mathrm{mg} / \mathrm{L}$, 不同湖泊水体溶解有机碳 (DOC) 浓度随富营养化程度等因素变化, 如贵州红枫湖水体中 DOC 浓度范围为 $1.60 \sim 3.08 \mathrm{mg} / \mathrm{L}$, 太湖水体 DOC 浓度随季节和点位不同在 $3 \sim 22 \mathrm{mg} / \mathrm{L}$ 范围内浮动, 巢湖水体的 DOC 浓度略低于太湖, 范围在 $2.85 \sim$ $12.04 \mathrm{mg} / \mathrm{L}$ 之间 ${ }^{[3]}$. 但之前对 DOM 的研究只关注其总的含量, 不同分子量 DOM 在湖泊水体中分布的有关 研究相对较少. 实际上, DOM 具有分子量连续分布特征, 可通过超滤技术将 DOM 分为低分子量 ( LMW, 分子 量 $<1 \mathrm{kDa}$ ) 和高分子量 ( HMW, 分子量 $1 \mathrm{kDa} \sim 0.45 \mu \mathrm{m}$ ) 部分, HMW-DOM 主要由腐殖质、蛋白质、氨基酸、多 糖和多酚等物质构成, LMW-DOM 则主要由有机酸、单糖和低聚糖等亲水性组分构成 ${ }^{[4]}$. 不同分子量 DOM 与污染物的结合程度不一样, 环境效应也不同. 一般认为 HMW-DOM 和 LMW-DOM 结合重金属的能力有所 不同, 有说法认为具有较高芳香度的大分子量 DOM 可能具有更多的重金属络合点 ${ }^{[5]}$, 但也有说法认为分子 量较小的小分子量 DOM 具有更大的比表面积, 从而拥有更强的重金属结合能力 ${ }^{[6]}$. 因此, DOM 作为环境中 众多有机、无机污染物的载体, 研究其分子量分布及苂光特征, 对了解水体中 DOM、各种元素的地球化学循 环, 从而进一步研究水体中污染物 (重金属、农药等) 的迁移、转化、生物有效性和归趋,有着重要意义 ${ }^{[2]}$.

鄱阳湖是我国第一大淡水湖, $97 \%$ 的流域面积处于江西省境内 $\left(28^{\circ} 25^{\prime} \sim 29^{\circ} 45^{\prime} \mathrm{N}, 115^{\circ} 48^{\prime} \sim 116^{\circ} 44^{\prime} \mathrm{E}\right.$, 图 1), 是周边农业灌溉、工业和生活用水的重要来源 ${ }^{[7]}$. 近年来, 随着流域内人口的增加、经济的发展, 尤其是 有色矿产资源的开发, 鄱阳湖的重金属污染现状不容乐观 ${ }^{[8-9]}$. 目前对于鄱阳湖 DOM 的研究较少, 且主要集 中于未分级 DOM 的 DOC 浓度、三维苂光光谱一平行因子分析及相关特性 ${ }^{[10]}$, 暂时未见鄱阳湖 DOM 不同分 子量分布、荧光特性及其对水体中重金属影响的研究. 有研究表明 DOM 可主要通过结构中的含氧官能团 (酚羟基、羒基等) 与水体中的一些重金属产生强烈的络合作用,如日本的 Sagami 河流中 DOM 与 $\mathrm{Cu}$ 和 $\mathrm{Fe}$ 之间的亲和力较强 ${ }^{[11]}$. 而不同水体 DOM 与重金属相关性的研究存在不确定性 ${ }^{[12]}$, 例如渤海小清河一莱州 湾 DOM 与 $\mathrm{Hg}$ 之间的相关性并不显著 ${ }^{[13]}$. 本文以鄱阳湖水体 DOM 为研究对象, 采用超滤技术进行 DOM 的 分级操作并采用平行因子分析法进行不同分子量荧光组分分析, 结合多种重金属元素在 LMW- - HMW-DOM 中的分布特征, 开展鄱阳湖水体中不同分子量 DOM 及其与重金属的关联性研究, 以期为湖区重金属污染防 治提供理论与技术支持.

\section{1 材料与方法}

\section{1 水样采集}

鄱阳湖具有典型的丰水期和枯水期特征, 每年的 4-9 月份一般为丰水期 ${ }^{[14]}$. 借助全球定位系统 (GPS) 于 2019 年春季 (4 月)在鄱阳湖 9 个样点采集水样(图 1), 在包含前人经典样点 (如吴城、鲤鱼洲等) 的基础 上进行大跨度采样. 使用采水器采集水样并润洗棕色取样瓶及瓶盖, 水样于 $24 \mathrm{~h}$ 内运回实验室后及时用 $0.45 \mu \mathrm{m} \mathrm{GF} / \mathrm{F}$ 玻璃纤维滤膜进行水样过滤, 并尽快完成超滤以及水质参数测定. 水样 $\mathrm{pH}$ 通过 $\mathrm{pH}$ 计进行测 定, 电导率通过电导率仪测定, 将电极探头伸人水样液面以下, 待示数稳定后读取并记录. 氨氮浓度的测定 采用纳氏试剂比色法, 硝态氮浓度的测定采用紫外分光光度法, 亚硝态氮浓度的测定采用 N-(1-荎基)-乙二 胺光度法, 磷酸盐浓度的测定采用钼蓝比色法. 


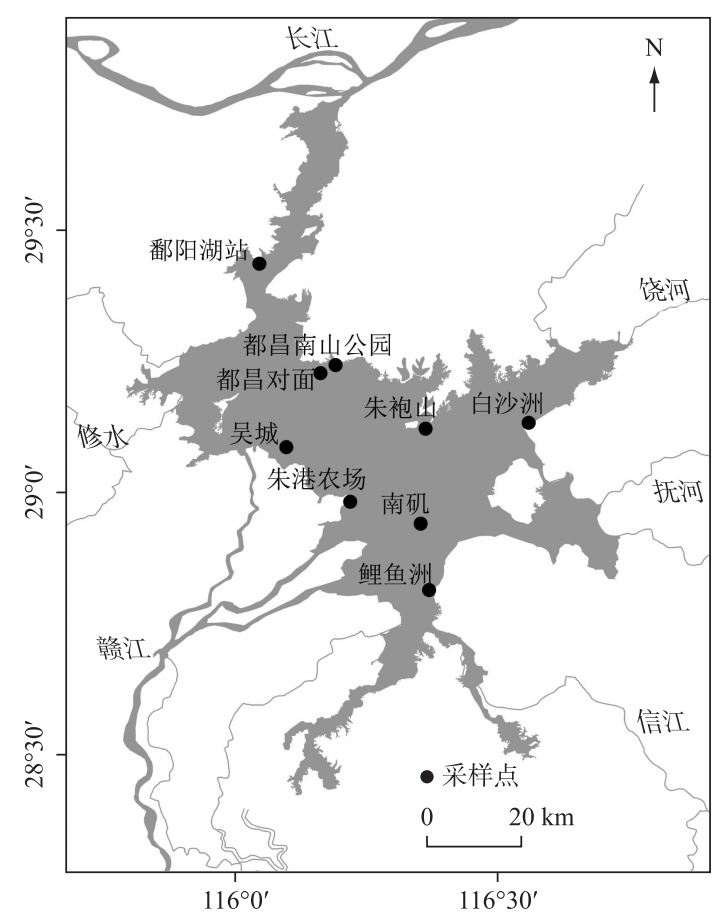

图 1 鄱阳湖采样点分布

Fig.1 Locations of sampling sites in Lake Poyang

\section{2 超滤系统及操作}

通过装有摚拌槽和 $1 \mathrm{kD}$ 孔径滤膜 (Millipore, PLAC07619) 的超过滤系统 (Amicon, USA) 将水样 DOM 分 为 LMW 组分 $(<1 \mathrm{kDa})$ 和 HMW 组分 $(1 \mathrm{kDa} \sim 0.45 \mu \mathrm{m})$, 透过超滤膜的超滤液为 LMW 组分, 而超滤杯中截 留液为 $\mathrm{HMW}$ 组分. 超滤前先用 $0.5 \mathrm{~mol} / \mathrm{L}$ 的 $\mathrm{NaOH}$ 溶液和大量的超纯水清洗超滤装置, 而后在 $220 \mathrm{kPa}$ 的 氮气压力以及 $150 \mathrm{r} / \mathrm{min}$ 的转速下进行超滤. 超滤装置用铝䈃纸包裹以避光. 单次超滤操作的体积浓缩因子 为 20 . 超滤回收率在 $105.1 \% \sim 122.5 \%$ 之间 (样品南矶除外), 平均为 $115.3 \%$.

\section{3 有机碳测定与光谱分析}

通过 TOC 分析仪 (Shimadzu, TOC-L) 测定各样点不同分子量 DOM 的 DOC 浓度. 使用紫外分光光度计 (UV-2550, Shimadzu) 测定吸收光谱, 比色血光路为 $10 \mathrm{~mm}$, 波长范围为 $200 \sim 800 \mathrm{~nm}$, 扫描步长为 $1 \mathrm{~nm}$. 吸收 系数 $\left(a(\lambda), \mathrm{m}^{-1}\right)$ 通过 $a(\lambda)=2.303 A(\lambda) / L$ 获得, 其中 $A(\lambda)$ 是样品在波长 $\lambda(\mathrm{nm})$ 处的吸光度, $L$ 为比色正. 光程 $(m)$. 紫外特征吸光度 (SUVA/SUVA 254 ) 被定义为 $A_{254}$ 与水样 DOC 浓度的比值, 其值越大, DOM 芳香化 程度越高 ${ }^{[15]}$. 光谱斜率 ( Slope $_{275-295}$ ), 也就是 $275 \sim 295 \mathrm{~nm}$ 处自然对数光谱的一阶导数, 采用非线性拟合公 式 $A(\lambda)=A\left(\lambda_{0}\right) \mathrm{e}^{S(\lambda 0-\lambda)}$ 获得, DOM 的分子量大小与 Slope $_{275-295}$ 的值呈反比 ${ }^{[16]}$.

\section{4 三维荧光光谱测定}

采用苂光分光光度计 (Hitachi F-7000) 测定水样的三维苂光光谱 (EEMs). 激发波长 (Ex) 和发射波长 (Em) 的区间分别为 200 450 和 250 550 nm, 增量分别为 $10 \mathrm{~nm}$ 和 $2 \mathrm{~nm}$, 扫描速度为 $2400 \mathrm{~nm} / \mathrm{min}$, PMT 电 压为 $700 \mathrm{~V}$. 以超纯水作为空白样并扣除瑞利和拉曼散射. 采用 Matlab 软件的 DOMFluor 工具箱对 27 个样 品进行平行因子模型解析 ${ }^{[17]}$, 通过残差分析、裂半分析以及目视检查验证正确的组分数 ${ }^{[18]}$. 采用的苂光参 数, 包括荧光指数 ( $F I X, \mathrm{Ex}=370 \mathrm{~nm}$ 时发射波长 $\mathrm{Em}$ 在 450 和 $500 \mathrm{~nm}$ 荧光强度的比率), 生物指数 ( $B I X$, $\mathrm{Ex}=310 \mathrm{~nm}$ 时发射波长 $\mathrm{Em}$ 在 380 和 $430 \mathrm{~nm}$ 苂光强度的比值) 和腐殖化指数 ( $H I X$, Ex $254 \mathrm{~nm}$ 处 $\mathrm{Em} 435 \sim$ $480 \mathrm{~nm}$ 区域积分值除以 $\mathrm{Em} 300 \sim 345 \mathrm{~nm}$ 与 $\mathrm{Em} 435 \sim 480 \mathrm{~nm}$ 区域积分值之和 $)^{[19-20]}$. 


\section{5 重金属元素测定}

通过电感耦合等离子质谱仪 (ICP-MS, Agilent 7500, 美国) 测定样品中 $\mathrm{Cr} 、 \mathrm{Mn} 、 \mathrm{Fe} 、 \mathrm{Co} 、 \mathrm{Ni} 、 \mathrm{Cu} 、 \mathrm{Zn} 、 \mathrm{As} 、 \mathrm{Se} 、$ $\mathrm{Cd} 、 \mathrm{Sb} 、 \mathrm{Ba} 、 \mathrm{~Pb}$ 的元素浓度. 水样的消解采用低浓度硝酸消解法. 为保证测定结果的准确性和精度, 测量过程 中对样品进行了加标回收实验 (回收率在 $90 \%$ 107\% 之间) 和重复分析 (重复率为 10\%), 所有样品检测结 果的分析误差均少于 $10 \%$,符合质量控制要求.

\section{2 结果与讨论}

\section{1 水样基础理化性质}

鄱阳湖各样点水体的基本理化指标可知, 鄱阳湖水体 $\mathrm{pH}$ 值均呈弱碱性 (7.67 7.82), 各点位间差异很 小 (表 1$)$. 总体来说, 平均总氮浓度 $(0.77 \mathrm{mg} / \mathrm{L})$ 和磷酸盐浓度 $(0.037 \mathrm{mg} / \mathrm{L})$ 与 $\mathrm{Yao}$ 等 $^{[7]}$ 以及杨桂山等 ${ }^{[21]}$ 测 得的数据基本相当. 另外, 西面 4 个点位 (鄱阳湖站、吴城、朱港水、南矶、鲤鱼洲) 总氮和磷酸根浓度的平均 值分别为 0.92 和 $0.051 \mathrm{mg} / \mathrm{L}$, 高于东部 4 个点位 (白沙洲、朱袍山、都昌南山、都昌对面) 的 0.59 和 $0.019 \mathrm{mg} / \mathrm{L}$, 与前人研究一致的西面氮磷浓度高于东面的分布特征一致 ${ }^{[22]}$, 也可能与西面的几个采样点 (如 吴城、鲤鱼洲) 位于河口附近, 而支流河水中氮磷浓度大于鄱阳湖水有关 ${ }^{[23]}$.

表 1 鄱阳湖各样点水样的理化指标

Tab.1 Physicochemical properties of each water sample site in Lake Poyang

\begin{tabular}{|c|c|c|c|c|c|c|}
\hline 采样点 & $\mathrm{pH}$ & 电导率/ $(\mu \mathrm{S} / \mathrm{cm})$ & 氨氮/(mg/L) & 硝态氮/ $(\mathrm{mg} / \mathrm{L})$ & 亚硝态氮/ $(\mathrm{mg} / \mathrm{L})$ & 磷酸盐/ $(\mathrm{mg} / \mathrm{L})$ \\
\hline 鄱阳湖站 & 7.67 & 108.1 & 0.196 & 0.775 & 0.022 & 0.045 \\
\hline 吴城 & 7.77 & 95.9 & 0.167 & 0.746 & - & 0.051 \\
\hline 朱港农场 & 7.82 & 95.7 & 0.155 & 0.754 & 0.009 & 0.042 \\
\hline 南矶 & 7.81 & 102.7 & 0.161 & 0.635 & 0.016 & 0.068 \\
\hline 鲤鱼洲 & 7.74 & 92.7 & 0.225 & 0.699 & 0.028 & 0.049 \\
\hline 白沙洲 & 7.81 & 154.0 & 0.173 & 0.490 & 0.005 & 0.021 \\
\hline 朱袍山 & 7.74 & 100.3 & 0.150 & 0.223 & 0.0004 & 0.014 \\
\hline 都昌南山 & 7.46 & 50.7 & 0.249 & 0.181 & 0.000 & 0.010 \\
\hline 都昌对面 & 7.72 & 129.9 & 0.249 & 0.632 & 0.009 & 0.033 \\
\hline
\end{tabular}

“一”表示未测出.

\section{$2.2 \mathrm{DOC}$ 分布特征}

鄱阳湖各点位的 DOC 浓度在 $1.54 \sim 2.64 \mathrm{mg} / \mathrm{L}$ 之间 (图 2a), 平均浓度 $1.95 \pm 0.33 \mathrm{mg} / \mathrm{L}$ 略高于 Yao 等 ${ }^{[7]}$ 于 2015 年 6 月份采样测得的 $1.63 \mathrm{mg} / \mathrm{L}$. TOC 值较低可能与丰水期大量输人的水其稀释作用有关. 湖内 DOC 浓 度分布规律与肖远东等 ${ }^{[24]}$ 的研究相似, 鄱阳湖站、都昌南山以及都昌对面 3 个点位的 DOC 浓度明显高于其他 点位. 各点位水体 DOM 分子量分布如图 2b 所示, 大部分采样点的 HMW 组分所占比例都在 $45 \% \sim 48 \%$ 之间, 而都昌南山和都昌对面两个点位水样的 HMW 组分所占比例高达 $57 \%$,可能的原因分析见 2.3 章节.

\section{3 光谱特征}

2.3.1 DOM 荧光特征 图 3 是朱袍山样点不同分子量 DOM 的三维苂光光谱. “寻峰” ${ }^{[25]}$ 后可见, 紫外区类腐 殖峰 A (230 260/380 460 nm) 主要分布在 LMW-DOM 中, 可见光区类腐殖峰 C $(320 \sim 360 / 420 \sim 480 \mathrm{~nm})$ 在 LMW-DOM 和 HMW-DOM 中都有分布, 类蛋白 (类色氨酸) 菼光峰 T( S 峰) (225 230/345 350 nm) 则主要 存在于 HMW-DOM. 此外, 对都昌对面和都昌南山这两个点位 HMW-DOM 的三维苂光图谱(篇幅原因, 文中 未给出) 与菼光强度进行观察和分析, 发现其中类蛋白峰 $\mathrm{T}$ 峰的强度远远高于鄱阳湖其他点位, 高 $\mathrm{T}$ 峰强度 可能解释了这 2 个点位的高 HMW-DOM 比例.

2.3.2 EEM-PARAFAC 三维荧光一平行因子分析 通过 PARAFAC 平行因子分析对 27 个样品的三维苂光谱 图进行解谱, 折半检验后确定了样品中 DOM 的 3 种主要苂光组分 (图 4), 包括类富里酸苂光组分 C1、C2 和 类腐殖酸苂光组分 $\mathrm{C} 3^{[26]} . \mathrm{C} 1$ 组分 $(\mathrm{Ex}=235 \mathrm{~nm}(300 \mathrm{~nm}), \mathrm{Em}=395 \mathrm{~nm})$ 属于短波类腐殖苂光组分, 以富 

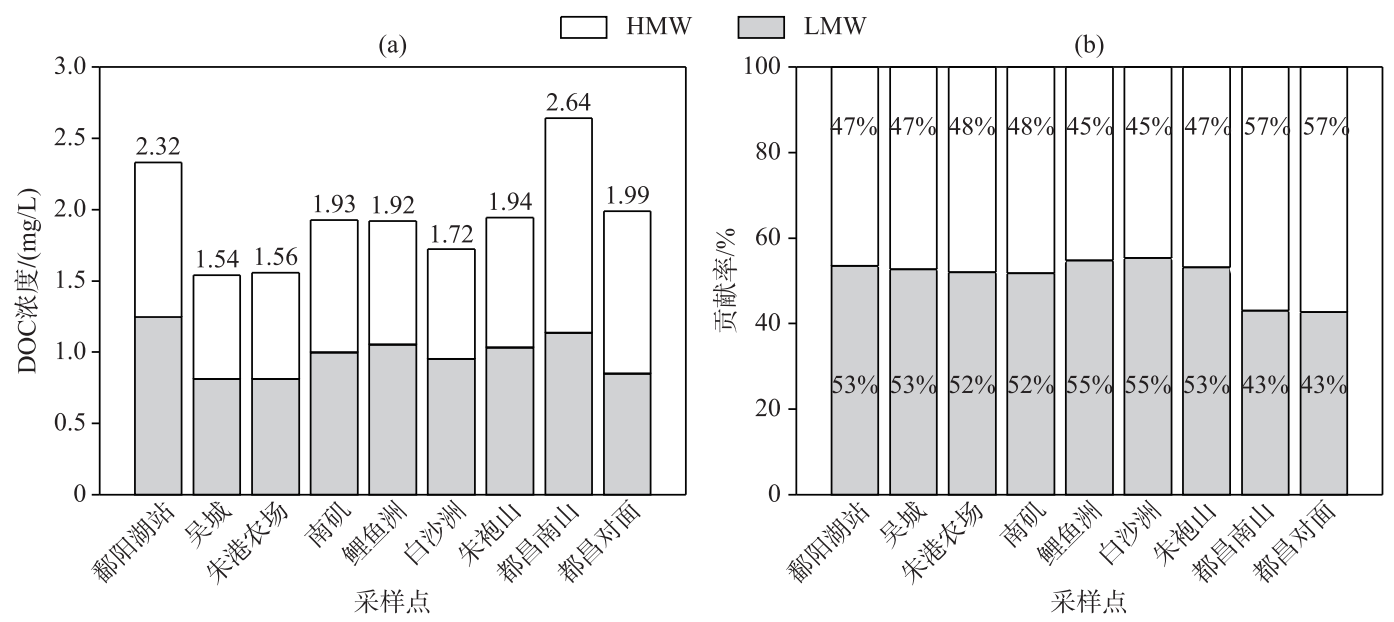

图 2 鄱阳湖各点位水样 DOC 浓度 (a) 及 LMW、HMW 组分对 DOC 值的贡献 (b)

Fig.2 DOC concentration of each water sample site in Lake Poyang (a) and contributions of LMW and HMW components (b)
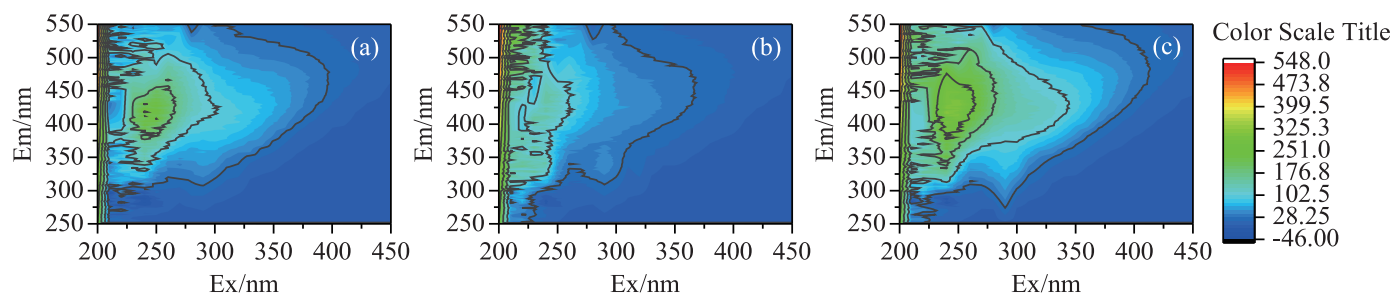

图 3 鄱阳湖水体不同分子量 DOM 的典型三维荧光光谱

(以朱袍山为例, a: LMW-DOM,b: HMW-DOM,c: bulk DOM )

Fig.3 Typical three-dimensional fluorescence spectra of DOM with different molecular weights in Lake Poyang

(Taking Zhupaoshan as an example,a: LMW-DOM,b: HMW-DOM,c: bulk DOM)

里酸为代表, 具有 2 个激发峰和 1 个发射峰, 其中 $235 / 395 \mathrm{~nm}$ 峰位于传统的 A 峰区域, 300/395 nm 峰位于 传统的海洋类腐殖荧光峰 M 峰 $(290 \sim 310 / 370 \sim 420 \mathrm{~nm})$ 区域 ${ }^{[27]}$. C2 组分 $(\mathrm{Ex}=200 \mathrm{~nm}, \mathrm{Em}=330 \mathrm{~nm}(450$ $\mathrm{nm})$ ) 具有 1 个激发峰和 2 个发射峰, 其中 200/450 nm 峰对应于传统的 A 峰区域, 200/330 nm 峰对应于传 统的 $\mathrm{T}$ 峰区域, 代表类蛋白质 (类色氨酸)苂光峰 ${ }^{[27]}$. C3 组分 $(\mathrm{Ex}=255 \mathrm{~nm}(360 \mathrm{~nm}), \mathrm{Em}=470 \mathrm{~nm}$ ) 属于长 波类腐殖质, 以腐殖酸为代表, 相对于 $\mathrm{C} 1$ 而言, 更长的激发和发射峰揭示了 $\mathrm{C} 3$ 的更大分子量, 被认为是传 统陆源类腐殖峰 $\mathrm{A}$ 和 $\mathrm{C}$ 的组合. 其中, C1、C3 的激发波长、发射波长与 Ishii 等 ${ }^{[28]}$ 的研究一致, C1、C2 的激发 波长与蔡文良等 ${ }^{[26]}$ 的研究结果相比发生了蓝移,发射波长发生了红移.

2.3.3 不同分子量 DOM 中荧光组分的分布特征 3 种荧光组分 $\mathrm{C} 1 、 \mathrm{C} 2 、 \mathrm{C} 3$ 在水样中所占比例为 $\mathrm{C} 1>\mathrm{C} 2>\mathrm{C} 3$ (图 5a). 图 5 列出了各个点位水样 DOM 中 3 种荧光组分 $\mathrm{C} 1$ (图 5b)、C2(图 5c)、C3(图 5d) 在不同分子量 DOM 中的分布: $60 \%$ 73\% 的 C1 组分分布在 LMW 组分中, $67 \%$ 93\% 的 C2 组分分布在 HMW 组分中, C3 组 分在几乎平均分布在 LMW 、HMW 部分中. 3 种苂光组分的分子量分布特征可结合章节 2.3.2 中其各自包含 的传统荧光峰来分析, C 峰与 $\mathrm{M}$ 峰相比主要由一些难降解的大分子量物质组成, 而 $\mathrm{T}$ 峰代表大分子蛋白类 有机物, 因此就分子量而言, C2 $>$ C3 $>$ C1. Y Y a 等 ${ }^{[7]}$ 通过主成分分析发现 C2 组分 (主要分布于 HMW-DOM 中) 主要来源于长江来水的补给, 结合前文距长江较近几个点位 (如都昌对面、都昌南山) 的 DOC 浓度与分子量 分布, 长江来水补给的相对高 C2 组分可能是此处高 DOC 浓度与高 HMW-DOM 比例的原因. 
C1

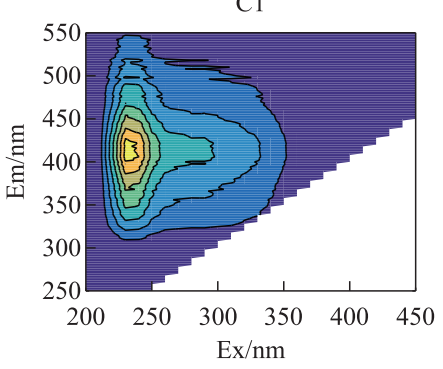

$\mathrm{C} 2$

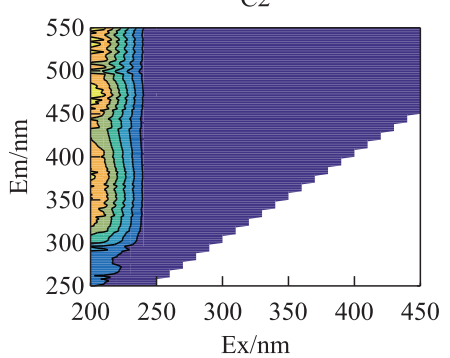

$\mathrm{C} 3$

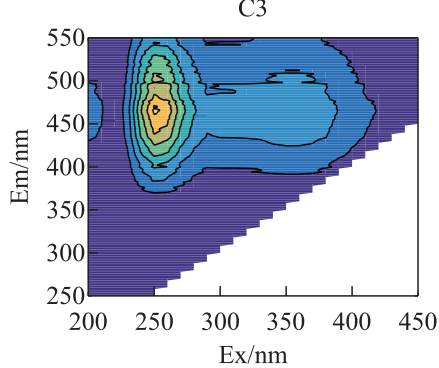

图 4 平行因子分析得到的鄱阳湖水样中 3 个苂光组分 (C1、C2 和 C3)

Fig.4 Three fluorescent components C1,C2 and C3 in Lake Poyang derived from EEM-PARAFAC

(a)

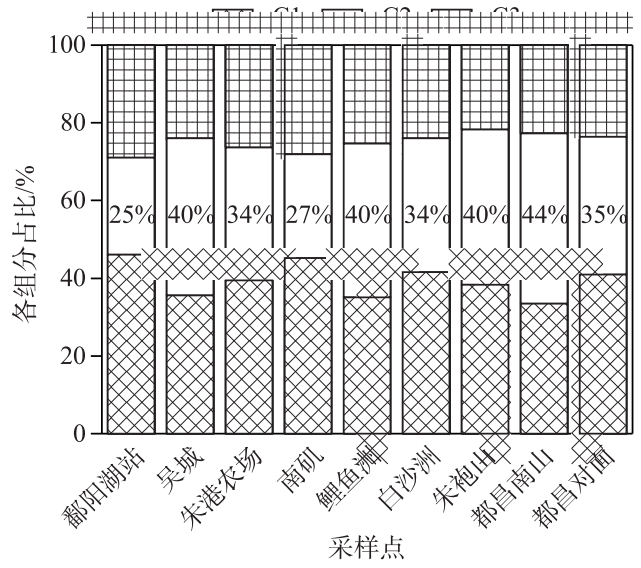

(c) $\mathrm{C} 2$

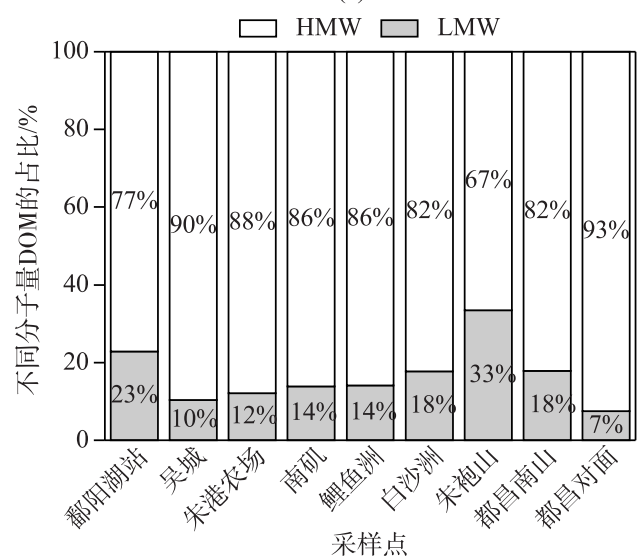

(b) $\mathrm{C} 1$

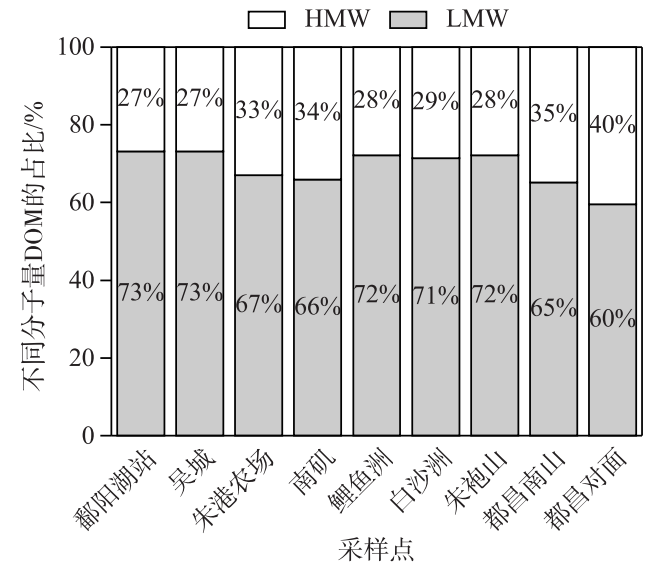

(d) $\mathrm{C} 3$

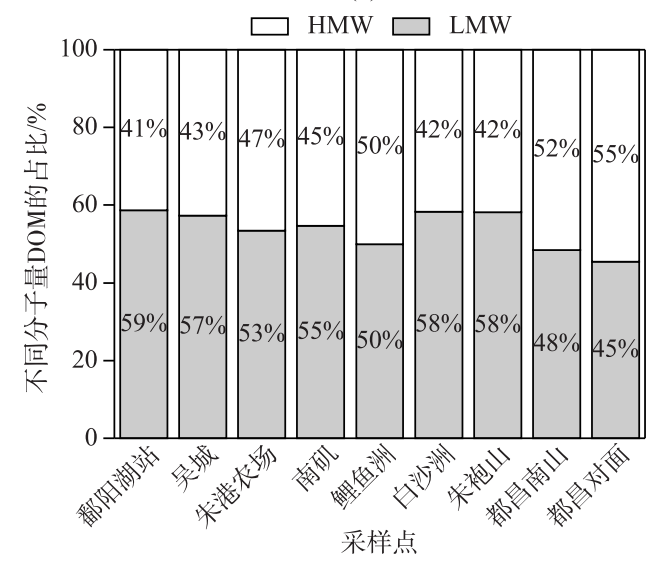

图 5 各点位水样中 3 个苂光组分所占比例 (a) 及在不同分子量 DOM 中的分布 $(b 、 c 、 d)$

Fig.5 Proportions of fluorescent components ( C1,C2 and C3) in each sample site (a) and distributions of fluorescent components among DOM with different molecular weights $(b, c, d)$

2.3.4 光谱参数 鄱阳湖 9 个样点水样的一些光谱参数值可见 (表 2), 每个样点 $\mathrm{SUVA}_{254}$ 最大的值均为 $\mathrm{HMW}$ 部分, 其次一般为未分级 DOM, 最后为 LMW 部分 (鄱阳湖站、白沙洲站、朱袍山站除外), 与其他文献中描述 的地表水体中 HMW 组分芳香性一般大于 LMW 组分的规律性一致 ${ }^{[29]}$; 各样点之间 LMW 组分的 SUVA 差别 不大, 吴城、朱港农场、鲤鱼洲、都昌对面、都昌南山的未分级 DOM 和 HMW 组分高于其他样点, 可能与河流 
输送的污染物有关, 说明水体芳香度主要受人为输人的控制.

表 2 鄱阳湖不同点位水样不同分子量的光谱参数

Tab.2 Spectral parameters of different molecular weights of water sample sites in Lake Poyang

\begin{tabular}{cccccccccccc}
\hline 光谱参数 & DOM & $\begin{array}{c}\text { 鄱阳 } \\
\text { 湖站 }\end{array}$ & 吴城 & $\begin{array}{c}\text { 朱港 } \\
\text { 农场 }\end{array}$ & 南矶 & 鲤鱼洲 & 白沙洲 & 朱袍山 & $\begin{array}{c}\text { 都昌 } \\
\text { 南山 }\end{array}$ & $\begin{array}{c}\text { 都昌 } \\
\text { 对面 }\end{array}$ \\
\hline $\mathrm{SUVA}_{254} /$ & $\mathrm{LMW}$ & 0.026 & 0.028 & 0.021 & 0.027 & 0.029 & 0.031 & 0.028 & 0.022 & 0.027 \\
$(\mathrm{~L} /(\mathrm{mg} \cdot \mathrm{m}))$ & $\mathrm{HMW}$ & 0.033 & 0.055 & 0.045 & 0.030 & 0.073 & 0.034 & 0.027 & 0.051 & 0.048 \\
& $\mathrm{DOM}$ & 0.026 & 0.046 & 0.037 & 0.028 & 0.052 & 0.031 & 0.022 & 0.048 & 0.045 \\
\multirow{2}{*}{$F I X$} & $\mathrm{LMW}$ & 1.38 & 1.35 & 1.40 & 1.38 & 1.37 & 1.41 & 1.31 & 1.41 & 1.38 \\
& $\mathrm{HMW}$ & 1.46 & 1.25 & 1.20 & 1.17 & 1.24 & 1.21 & 1.24 & 1.26 & 1.20 \\
& $\mathrm{DOM}$ & 1.27 & 1.30 & 1.34 & 1.30 & 1.30 & 1.31 & 1.27 & 1.23 & 1.29 \\
\multirow{2}{*}{$B I X$} & $\mathrm{LMW}$ & 0.78 & 0.77 & 0.78 & 0.81 & 0.79 & 0.81 & 0.86 & 0.77 & 0.79 \\
& $\mathrm{HMW}$ & 0.60 & 0.63 & 0.62 & 0.65 & 0.65 & 0.65 & 0.70 & 0.59 & 0.63 \\
& $\mathrm{DOM}$ & 0.70 & 0.70 & 0.68 & 0.70 & 0.72 & 0.73 & 0.76 & 0.66 & 0.72 \\
\hline
\end{tabular}

$F I X$ 反映了芳香族与非芳香族 DOM 的相对贡献, 总体而言与 DOM 的芳香度呈负相关 ${ }^{[30]}$, 可以提供关于 DOM 样品的来源或降解程度的信息. 陆源和微生物来源 DOM 的 FIX 值分别为 1.4 和 $1.9, F I X>1.9$ 时, 表示 DOM 来源主要以内源 (微生物活动、藻类活动) 为主, 自生源特征明显; $F I X<1.4$ 时, DOM 则主要源于陆源 (外 源输人 $)^{[30]}$. 鄱阳湖 9 个样点 FIX 值基本在 1.4 以下, 呈现显著的陆源特征, 与 Yan 等的研究结果一致 ${ }^{[31]}$; 除 鄱阳湖站外, 各点位水样中 LMW 组分的 FIX 值均大于 HMW 组分, 说明内源(微生物来源) 的基团主要分布在 LMW 组分中, 而陆源 DOM 主要分布在 HMW 组分中, 与先前研究结果一致 ${ }^{[32]}$. BIX 值作为 DOM 来源的指示 参数 ${ }^{[33]}$, 用于确定 $\beta$ 苂光团的存在, 这是表示水样中原生生物活性的特征. $B I X$ 值可用于评估样本中微生物来 源的 DOM 的相对贡献率 ${ }^{[34]}$, 高的 $B I X$ 值指示样品中较高的自生源贡献率, 低的 $B I X$ 值指示人为来源. $B I X$ 值 基本处于 $0.6 \sim 0.8$ 之间, 表明自生源贡献的 DOM 较少 ${ }^{[19]}$; LMW-DOM 的 BIX 值大于 HMW-DOM 和 bulk DOM, 说明各点位的 LMW 部分具有较强新生自生源特征, 自生源 DOM 主要分布在 LMW 组分. 各点位之间 FIX 值和 $B I X$ 值差别不大, 但鄱阳湖站、都昌南山、都昌对面 3 处的 $F I X$ 和 $B I X$ 值均略小于其他样点, 说明陆源 DOM 比 例高于别处; 结合 2.2 节 DOC 分布特征中这 3 处 DOC 和 HMW-DOM 比例偏高的特征, 表明这 3 个样点附近可 能有高于其他点位的人为干扰, 可能与丰水期长江来水倒灌鄱阳湖的现象有关 ${ }^{[7]}$.

\section{4 重金属元素浓度及分布}

从各点位未进行分级水样中重金属元素浓度来 看, 鄱阳湖水体重金属浓度排序为: $\mathrm{Zn}>\mathrm{Cu}>\mathrm{Pb}>\mathrm{Cr}>$ $\mathrm{Cd}$,与其他文献测定的土壤/底泥中重金属浓度排序 $(\mathrm{Zn}>\mathrm{Pb}>\mathrm{Cr}>\mathrm{Cu}>\mathrm{Cd})$ 相比 ${ }^{[8]}$, $\mathrm{Cu}$ 在水样中具有较高 的浓度. 其原因可能与 $\mathrm{Cu}$ 对 DOM 较强的络合能力 有关, 已有研究通过 EEM-PARAFAC 技术发现 $\mathrm{Cu}$ 与 DOM 存在较好的荧光淬灭现象, 证明 $\mathrm{Cu}$ 与 DOM 之 间相互作用较强 ${ }^{[35]}$.

通过配对样本均值检验 ( pair-sample $t$-test) 对 LMW-DOM 和 HMW-DOM 中的重金属浓度高低进行 比较 (表 3), 除 $\mathrm{Cr} 、 \mathrm{Co} 、 \mathrm{Cu} 、 \mathrm{Zn}$ 这几种重金属在 LMW 和 HMW 组分中含量在 0.05 的显著性水平上无显著 差异外,其余元素在不同分子量 DOM 中的分布差异 显著, 置信度基本达到 $95 \%$ 以上 ( 除了 $\mathrm{Cd}$ 为 $93 \%$ ). 结合图 6 重金属元素在鄱阳湖 LMW-DOM、HMW-

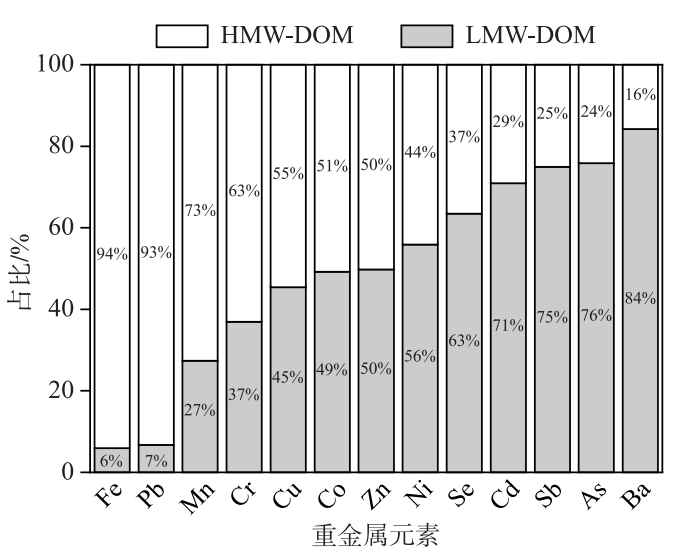

图 6 各种重金属元素在鄱阳湖 LMW-DOM、 HMW-DOM 中的占比

Fig.6 Percentage of various heavy metal elements in LMW-DOM and HMW-DOM of Lake Poyang 
DOM 中的占比, 发现 Co、Ni、Cu、Zn 几种元素在 LMW 、HMW 组分中的分布基本相当( 与不同分子量中分布 差异不显著的样本均值检验结果相符); $\mathrm{Se}(63 \%) 、 \mathrm{Cd}(71 \%) 、 \mathrm{Sb}(75 \%) 、 \operatorname{As}(76 \%) 、 \mathrm{Ba}(84 \%)$ 主要分布在 LMW 组分, $\operatorname{Cr}(63 \%) 、 \operatorname{Mn}(73 \%)$ 大部分分布在 HMW 组分, $\mathrm{Fe} 、 \mathrm{~Pb}$ 则绝大部分 $(>90 \%)$ 分布在 $\mathrm{HMW}$ 组分. $\mathrm{Fe}$ 主要分布在 HMW-DOM 中可能是由于主要分布在 LMW-DOM 中的 C1 对 $\mathrm{Fe}$ 的络合能力最低 ${ }^{[28]} ; \mathrm{Pb}$ 的分 布则与其他文献中与分子量小于 $0.5 \mathrm{ku}$ 的 DOM 结合系数最高的规律有所出 ${ }^{[36]}$. 由各种重金属在不同分 子量 DOM 中的分布可见主要分布在 LMW 组分中的重金属元素种类较多, 但由于主要分布在 HMW 组分中 的重金属 $(\mathrm{Fe} 、 \mathrm{~Pb})$ 浓度高于其他元素, 因此 HMW-DOM 中的重金属 $(0.080 \mathrm{mg} / \mathrm{L})$ 大于 LMW-DOM $(0.046$ $\mathrm{mg} / \mathrm{L})$. 由此可见 DOM 与重金属的结合能力不能一概而论, 需要结合不同的重金属种类分别进行讨论, 可 在今后的研究中通过荧光淬灭等手段进行探讨.

表 3 各种重金属元素在鄱阳湖 LMW-DOM、HMW-DOM 中的分布 (单位: $\left.\times 10^{-2} \mu \mathrm{g} / \mathrm{L}\right)$ 及均值检验 $P$ 值

Tab.3 Distribution of various heavy metal elements (Unit: $\times 10^{-2} \mu \mathrm{g} / \mathrm{L}$ )

in LMW-DOM and HMW-DOM of Lake Poyang and $P$ values of pair-sample $t$-test

\begin{tabular}{|c|c|c|c|c|c|c|c|c|c|c|c|}
\hline 重金属 & DOM & $\begin{array}{l}\text { 鄱阳 } \\
\text { 湖站 }\end{array}$ & 吴城 & $\begin{array}{l}\text { 朱港 } \\
\text { 农场 }\end{array}$ & 南矶 & $\begin{array}{c}\text { 鲤鱼 } \\
\text { 洲 }\end{array}$ & $\begin{array}{c}\text { 白沙 } \\
\text { 洲 }\end{array}$ & $\begin{array}{c}\text { 朱袍 } \\
山\end{array}$ & $\begin{array}{l}\text { 都昌 } \\
\text { 南山 }\end{array}$ & $\begin{array}{l}\text { 都昌 } \\
\text { 对面 }\end{array}$ & $P$ 值 \\
\hline \multirow[t]{2}{*}{$\mathrm{Cr}$} & LMW & 7.39 & 8.99 & 8.77 & 5.91 & 9.18 & 15.58 & 6.51 & 10.43 & 14.47 & 0.158 \\
\hline & HMW & 3.92 & 11.69 & 5.93 & 12.45 & 37.69 & 12.15 & 14.26 & 39.74 & 11.18 & \\
\hline \multirow[t]{2}{*}{$\mathrm{Mn}$} & LMW & 14.60 & 3.32 & 18.32 & 4.27 & 4.69 & 40.37 & 14.53 & 52.45 & 14.50 & 0.027 \\
\hline & HMW & 0.39 & 56.35 & 15.75 & 50.97 & 103.77 & 57.71 & 26.14 & 73.87 & 58.88 & \\
\hline \multirow[t]{2}{*}{$\mathrm{Fe}$} & LMW & 225.9 & 231.3 & 241.6 & 248.8 & 1012.5 & 286.1 & 259.1 & 1063.1 & 215.4 & 0.039 \\
\hline & HMW & 973 & 5815 & 1955 & 2819 & 16659 & 2116 & 2034 & 23469 & 3788 & \\
\hline \multirow[t]{2}{*}{ Co } & LMW & 3.59 & 3.20 & 3.52 & 3.20 & 3.76 & 5.04 & 2.39 & 5.15 & 5.41 & 0.867 \\
\hline & HMW & 1.98 & 4.06 & 2.45 & 4.45 & 8.73 & 4.50 & 1.43 & 5.37 & 3.39 & \\
\hline \multirow[t]{2}{*}{$\mathrm{Ni}$} & LMW & 38.79 & 34.59 & 35.11 & 37.70 & 38.56 & 50.24 & 28.31 & 44.99 & 33.83 & 0.038 \\
\hline & HMW & 22.09 & 25.86 & 22.66 & 30.02 & 37.60 & 28.23 & 15.65 & 55.78 & 32.17 & \\
\hline \multirow[t]{2}{*}{$\mathrm{Cu}$} & LMW & 80.34 & 70.24 & 66.29 & 58.55 & 74.91 & 88.87 & 86.24 & 44.87 & 58.44 & 0.172 \\
\hline & HMW & 82.37 & 70.09 & 71.13 & 77.03 & 81.99 & 83.92 & 69.59 & 121.04 & 98.24 & \\
\hline \multirow[t]{2}{*}{$\mathrm{Zn}$} & LMW & 542 & 440 & 475 & 590 & 381 & 716 & 626 & 577 & 143 & 0.966 \\
\hline & HMW & 256 & 654 & 296 & 787 & 261 & 598 & 214 & 803 & 661 & \\
\hline \multirow[t]{2}{*}{ As } & LMW & 117.52 & 116.02 & 118.43 & 109.54 & 105.08 & 97.30 & 82.31 & 43.11 & 98.40 & $<0.001$ \\
\hline & HMW & 22.71 & 35.17 & 37.87 & 36.43 & 42.60 & 24.97 & 22.67 & 24.98 & 35.65 & \\
\hline \multirow[t]{2}{*}{$\mathrm{Se}$} & LMW & 0.71 & 0.68 & 0.78 & 0.76 & 0.94 & 1.08 & 1.21 & 0.38 & 1.16 & 0.002 \\
\hline & HMW & 0.33 & 0.40 & 0.49 & 0.49 & 0.65 & 0.40 & 0.67 & 0.49 & 0.52 & \\
\hline \multirow[t]{2}{*}{$\mathrm{Cd}$} & LMW & 1.27 & 1.45 & 1.42 & 1.48 & 2.05 & 2.13 & 1.53 & 3.42 & 7.25 & 0.062 \\
\hline & HMW & 0.31 & 1.77 & 0.65 & 1.18 & 1.69 & 1.34 & 0.27 & 0.90 & 0.90 & \\
\hline \multirow[t]{2}{*}{$\mathrm{Sb}$} & LMW & 23.29 & 16.80 & 15.05 & 15.85 & 17.29 & 27.43 & 22.16 & 17.76 & 21.88 & 0.002 \\
\hline & HMW & 3.31 & 24.03 & 2.33 & 3.42 & 3.87 & 4.90 & 3.26 & 9.38 & 4.81 & \\
\hline \multirow[t]{2}{*}{$\mathrm{Ba}$} & LMW & 4135 & 3584 & 3598 & 4172 & 3559 & 4125 & 3092 & 1796 & 2480 & $<0.001$ \\
\hline & HMW & 463 & 503 & 593 & 764 & 693 & 645 & 626 & 760 & 666 & \\
\hline \multirow[t]{2}{*}{$\mathrm{Pb}$} & LMW & 2.14 & 1.53 & 2.18 & 1.23 & 4.51 & 1.60 & 1.25 & 8.88 & 1.99 & 0.001 \\
\hline & HMW & 15.96 & 45.04 & 19.18 & 34.28 & 47.40 & 65.46 & 16.31 & 84.13 & 21.84 & \\
\hline
\end{tabular}

主要分布在 HMW 组分的 4 种重金属元素 $\mathrm{Fe} 、 \mathrm{~Pb} 、 \mathrm{Mn} 、 \mathrm{Cr}$ 中, $\mathrm{Fe} 94 \%$ 分布在 $\mathrm{HMW}$ 组分, 与长江河口的 $86.19 \%{ }^{[37]}$ 、美国新泽西 mullica 河的 $80 \%{ }^{[38]}$ 以及意大利 Venice Lagoon 的 $87.4 \%{ }^{\left[{ }^{[39]}\right.}$ 接近, 与其他人的研究共 同呈现出绝大部分分布在 HMW 组分的规律; Pb 在 HMW 组分中分布占全量的 $93 \%$, 与美国新泽西 mullica 河 $(88 \%)^{[38]}$ 接近,符合其他文献中 $\mathrm{Pb}$ 以胶体态为主 $(12 \% \sim 100 \%)$ 的结论 ${ }^{[40]}$; HMW 组分中 $\mathrm{Mn}$ 占全量的 $73 \%$, 高于长江口 $(60.63 \%)^{[32]}$ 和意大利 Venice Lagoon $(54 \%)^{[39]} ; \mathrm{Cr} 63 \%$ 分布在 HMW 组分中, 高于长江口 $(42.27 \%)^{[37]}$. 其余重金属元素中, Cu 55\% 分布在 HMW-DOM 中, 与长江河口 $(67.1 \%)^{[37]}$ 和英国梅德韦河 
(53\%) 类似 ${ }^{[41]}$, 但明显高于太湖 $(22.3 \%)^{[42]}$. Zn 50\% 分布在 HMW 组分, 略低于长江口 $(58.91 \%)^{[37]}$. Ni 在 HMW 组分分布平均占总量的 $44 \%$, 低于长江口的 $53.14 \%^{[37]}$, 但总体来说在 HMW 与 LMW 中的分布相差不 大,与前人研究一致 ${ }^{[43]}$. As $24 \%$ 分布在 HMW 组分, 低于长江河口的 $59.43 \%{ }^{[37]}$.

计算各种金属元素在 LMW-DOM 和 HMW-DOM 中的浓度标准差, 除 As、Se、Cd、Ba 外, 其余重金属在 HMW-DOM 中浓度的标准差均大于其在 LMW-DOM 中的标准差,说明鄱阳湖区水体中重金属元素的差异性 主要存在于 HMW-DOM 中. 结合前面 2.3 节部分采用平行因子分析得出的陆源 DOM 主要分布在 HMW 组分 中的结论, 说明鄱阳湖区水体重金属含量的分异主要受沿岸土壤径流及人为输人 (工业污水和生活污水) 影响 $^{[44]}$.

\section{5 重金属浓度与各项指标相关性分析}

进一步研究发现,鄱阳湖水体重金属浓度与未分级 DOM 各项指标之间的相关性大多不显著 (表 4), 可 能是因为环境中重金属丰度不仅与 DOM 浓度和其与 DOM 相互作用的强弱有关, 也可能与上游排放和其他 环境因素有关 ${ }^{[45]}$. 表 4 中相关性显著 $(P<0.05)$ 的关系共 4 对, 其中 $\mathrm{Cr} 、 \mathrm{Cu}$ 与 $\mathrm{C} 3$ 组分占比呈中高度正相关 $(r>0.7), \mathrm{Cr}$ 与 $\mathrm{C} 2$ 组分占比呈中度正相关 $(0.5<r<0.7), \mathrm{Se}$ 与 $\mathrm{C} 1$ 组分占比呈中高度正相关 $(r>0.7)$. LMW 与 HMW-DOM 中重金属浓度与每项指标的相关性表格由于篇幅原因就不列出, 下文列出关键数据. LMWDOM 中相关性显著 $(P<0.05)$ 的关系仅 2 对, 分别为 $\operatorname{Co}(r=0.73, P=0.02)$ 和 $\mathrm{Ni}(r=0.80, P=0.01)$, 其浓度 与 $F I X$ 值呈中高度正相关. HMW-DOM 中显著 $(P<0.05)$ 的相关关系达 9 对, Cr、Mn、Fe、Co、Ni 与 $\mathrm{Slope}_{275-295}$ 呈中到高度负相关 $(-0.82<r<-0.69), \mathrm{Mn} 、 \mathrm{Fe} 、 \mathrm{Co}$ 与 SUVA 值呈中度正相关 $(0.65<r<0.8), \mathrm{Cu}$ 与 DOC 浓度呈 极显著的高度正相关 $(r>0.9, P<0.01)$. 相比 HMW-DOM 中的 Cu 而言, LMW-DOM 中 Cu 与 DOC 浓度的 $r<$ 0.1 且显著性极差 $(P=0.83)$, 说明 HMW-DOM 与 $\mathrm{Cu}$ 的结合能力较强, 与石陶然等 ${ }^{[46]}$ 的研究结果一致.

表 4 鄱阳湖水体重金属浓度与未分级 DOM 各项指标的相关性分析

Tab.4 Correlation analysis between the heavy metal concentration and various indexes of bulk DOM in Lake Poyang

\begin{tabular}{crrrrrrrrr}
\hline 重金属 & DOC & \multicolumn{1}{c}{ BIX } & \multicolumn{1}{c}{$F I X$} & \multicolumn{1}{c}{$H I X$} & SUVA & Slope $_{275-295}$ & C1 占比 & C2 占比 & C3 占比 \\
\hline $\mathrm{Cr}$ & -0.37 & -0.30 & 0.50 & -0.54 & -0.45 & 0.35 & 0.55 & $-0.67^{*}$ & $0.76^{*}$ \\
$\mathrm{Mn}$ & -0.29 & -0.26 & 0.63 & -0.30 & -0.07 & 0.25 & 0.26 & -0.44 & 0.66 \\
$\mathrm{Fe}$ & -0.48 & -0.25 & 0.53 & -0.36 & 0.07 & -0.01 & -0.06 & -0.05 & 0.22 \\
$\mathrm{Co}$ & -0.30 & -0.49 & 0.46 & -0.21 & -0.08 & 0.10 & 0.02 & -0.18 & 0.43 \\
$\mathrm{Ni}$ & -0.12 & -0.39 & 0.12 & -0.21 & -0.04 & -0.03 & 0.09 & -0.29 & 0.61 \\
$\mathrm{Cu}$ & 0.09 & -0.49 & -0.11 & -0.68 & -0.18 & -0.06 & 0.45 & -0.59 & $0.72^{*}$ \\
$\mathrm{Zn}$ & -0.10 & -0.25 & 0.17 & -0.35 & 0.18 & -0.13 & 0.03 & -0.19 & 0.45 \\
$\mathrm{As}$ & 0.21 & 0.14 & -0.14 & 0.32 & -0.46 & 0.45 & 0.13 & -0.01 & -0.20 \\
$\mathrm{Se}$ & 0.38 & -0.04 & -0.27 & -0.40 & -0.41 & 0.21 & $0.73 *$ & -0.75 & 0.62 \\
$\mathrm{Cd}$ & -0.13 & -0.03 & 0.18 & 0.29 & 0.49 & -0.36 & -0.17 & 0.02 & 0.27 \\
$\mathrm{Sb}$ & 0.36 & -0.46 & -0.41 & -0.35 & 0.15 & -0.39 & 0.29 & -0.44 & 0.63 \\
$\mathrm{Ba}$ & -0.03 & -0.13 & -0.10 & 0.23 & -0.14 & -0.05 & 0.00 & 0.05 & -0.14 \\
$\mathrm{~Pb}$ & -0.60 & -0.20 & 0.45 & -0.29 & -0.15 & 0.04 & 0.22 & -0.29 & 0.37 \\
\hline $\mathrm{P}$ (0.05
\end{tabular}

$* P<0.05, n=9$.

以上为 LMW-DOM、HMW-DOM、bulk DOM 中各种重金属浓度与各项指标的讨论. 3 种 DOM 之间进行总 体比较的话, 发现 HMW-DOM 溶液中的重金属浓度与各项指标的皮尔森相关系数 $r(0.36)$ 高于 LMW-DOM $(0.30)$ 和 bulk $\operatorname{DOM}(0.29)$ 溶液, 且总体平均 $P$ 值 (0.42) 也低于 $\operatorname{LMW}-\operatorname{DOM}(0.48)$ 和 bulk $\operatorname{DOM}(0.50)$. 总体 而言, 重金属在 HMW-DOM 中与其他指标的相关系数与显著程度大于其他分子量 DOM. 结合 2.4 节中重金 属在 HMW 组分中标准差往往大于 LMW 组分的特征, 说明重金属离子在各采样点的分异主要受 HMW-DOM 的影响.

\section{3 结论}

1 ) 丰水期的鄱阳湖水体 DOC 浓度较低,平均为 $1.95 \pm 0.33 \mathrm{mg} / \mathrm{L}$. 鄱阳湖站、都昌南山以及都昌对面 3 个 
点位的 DOC 浓度高于其他点位, 其中都昌南山、都昌对面 2 个点位的 HMW-DOM 比例也显著高于其他点 位, 可能是丰水期长江来水倒灌鄱阳湖的原因. 总体来说, 鄱阳湖水体中 LMW 和 HMW 组分对 DOC 总量的 贡献基本相当.

2) 采用 EEM-PARAFAC 技术从水中 DOM 提取出 C1、C2、C3 三个类腐殖组分, 其中 C1 组分 $70 \%$ 分布在 LMW 组分中, C2 组分 $83 \%$ 分布在 HMW 组分中, C3 组分在不同分子量 DOM 中的分布基本相当, 可能与各 组分分别含有的传统苂光峰 M 峰、T 峰、C 峰的性质有关. LMW-DOM 具有更强的自生源特征, HMW-DOM 则 具有更强的陆源特征和更高的芳香性,说明水体 DOM 芳香度受人为输人的陆源 DOM 影响较大.

3) 探讨了各类重金属元素在鄱阳湖各点位 HMW-DOM 和 LMW-DOM 中的分布, 其中 $\mathrm{Se} 、 \mathrm{Cd} 、 \mathrm{Sb} 、 \mathrm{As} 、 \mathrm{Ba}$ 主要分布在 LMW-DOM 中, Co、Ni、Cu、Zn 元素在 LMW-DOM、HMW-DOM 中的分布基本相当, Cr、Mn 主要分 布在 $H M W$ 组分, $\mathrm{Fe} 、 \mathrm{~Pb}$ 则绝大部分分布在 $\mathrm{HMW}$ 组分.

4) HMW-DOM 溶液中的重金属浓度与各项指标的相关程度和显著性 $(r=0.36, P=0.42)$ 明显优于 $\operatorname{LMW}-\operatorname{DOM}(r=0.30, P=0.48)$ 和 bulk DOM $(r=0.29, P=0.50)$ 溶液, 且相关性显著的关系数量也高于 LMWDOM 和 bulk DOM,说明重金属离子在各采样点的分异主要与 HMW-DOM 性质相关.

\section{4 参考文献}

[ 1 ] Kitis M, Kilduff JE, Karanfil T. Isolation of dissolved organic matter ( DOM) from surface waters using reverse osmosis and its impact on the reactivity of DOM to formation and speciation of disinfection by-products. Water Research, 2001, 35(9) : 2225-2234. DOI: 10.1016/S0043-1354(00) 00509-1.

[ 2 ] Benoit G, Oktay-marshall SD, Cantu A et al. Partitioning of Cu, Pb, Ag, Zn, Fe, Al, and Mn between filter-retained particles, colloids, and solution in six texas estuaries. Marine Chemistry, 1994, 45(4) : 307-336. DOI: 10.1016/03044203( 94) 90076-0.

[ 3 ] Yang DT, Chen WM. The temporal and spatial distribution of dissolved organic carbon in lakes at the lower reach of Changjiang River. Environmental Pollution Control, 2004, 26(4) : 275-277. [杨顶田, 陈伟民. 长江下游湖泊中可溶 性有机碳的时空分布. 环境污染与防治, 2004, 26(4) : 275-277.]

[ 4 ] Gong XY, Zhang HZ, Hou C et al. Fluorescence excitation-emission matrix spectra coupled with physic and mathematic isolation to study composition of dissolved organic matter. Chinese Journal of Analytical Chemistry, 2016, 44(10) : 15331538. [ 巩学勇, 张宏志, 侯超等. 三维苂光光谱结合物理和数学分离研究溶解性有机质组成. 分析化学, 2016,44 (10) : 1533-1538.]

[ 5 ] Ramamoorthy S, Kushner DJ. Heavy metal binding sites in river water. Nature, 1975, 256(5516) : 399-401. DOI: 10. 1038/256399a0.

[ 6 ] Zarruk KKD, Scholer G, Dudal Y. Fluorescence fingerprints and $\mathrm{Cu}^{2+}$-complexing ability of individual molecular size fractions in soil- and waste-borne DOM. Chemosphere, 2007, 69(4) : 540-548. DOI: 10.1016/j.chemosphere.2007.03.039.

[ 7 ] Yao X, Wang SR, Ni ZK et al. The response of water quality variation in Lake Poyang (Jiangxi, People's Republic of China) to hydrological changes using historical data and DOM fluorescence. Environmental Science and Pollution Research, 2015, 22 (4) : 3032-3042. DOI: 10.1007/s11356-014-3579-z.

[ 8 ] Gong XF, Chen CL, Zhou WB et al. Assessment on heavy metal pollution in the sediment of Lake Poyang. Environmental Science, 2006, (4) : 732-736. DOI: 10.13227/j.hjkx.2006.04.026. [弓晓峰, 陈春丽, 周文斌等. 鄱阳湖底泥中重金 属污染现状评价. 环境科学, 2006, 27(4): 732-736.]

[ 9 ] Yang QY, Zeng M, Xie Q et al. The distribution of heavy metals and potential ecological risk in the Northern Lake Poyang. Ecology and Environmental Sciences, 27(3): 556-564. [杨期勇, 曾明, 谢琪等. 鄱阳湖北部湖区沉积物重金属分布 及其潜在生态风险评价. 生态环境学报, 2018, 27(3) : 556-564.]

[11] Kikuchi T, Fujii M, Terao K et al. Correlations between aromaticity of dissolved organic matter and trace metal concentrations in natural and effluent waters: a case study in the Sagami River Basin, Japan. Science of the Total Environment, 2017, 576: 36-45. DOI: 10.1016/j. scitotenv.2016.10.068.

[12] Liang MQ, Shao ML, Cao ML et al. Characteristics of dissolved organic matter (DOM) and relationship with dissolved heavy metals in a peri-urban and an urban river. Environmental Science, 2018, 39(5) : 2095-2103. DOI: 10.13227/j. hjkx.201710089. [梁梦琦, 邵美玲, 曹昌丽等. 城郊与城镇河流中溶解性有机质与重金属的相关性. 环境科学, 2018, 39(5) : 2095-2103.] 
[13] Jiang T, Skyllberg U, Björn E et al. Characteristics of dissolved organic matter ( DOM) and relationship with dissolved mercury in Xiaoqing River-Laizhou Bay estuary, Bohai Sea, China. Environmental Pollution, 2017, 223 : 19-30. DOI: 10. 1016/j.envpol.2016.12.006.

[14] Liu Y, Yu ZQ, Fan JP et al. The characters of backscattering coefficient during flood period in Lake Poyang. Journal of Central China Normal University: Natural Sciences, 2019, 53(2) : 283-289. [刘瑶, 余自强, 范杰平等. 鄱阳湖丰水期 水体后向散射特性研究. 华中师范大学学报: 自然科学版, 2019, 53(2) : 283-289.]

[15] Weishaar JL, Aiken GR, Bergamaschi BA et al. Evaluation of specific ultraviolet absorbance as an indicator of the chemical composition and reactivity of dissolved organic carbon. Environmental Sciences \& Technology, 2003, 37 (20) : 47024708. DOI: $10.1021 / \mathrm{es} 030360 x$.

[16] Helms JR, Stubbins A, Ritchie JD et al. Absorption spectral slopes and slope ratios as indicators of molecular weight, source, and photobleaching of chromophoric dissolved organic matter. Limnology and Oceanography, 2008, 53(3): 955969. DOI: 10.4319/lo.2008.53.3.0955.

[17] Stedmon CA, Bro R. Characterizing dissolved organic matter fluorescence with parallel factor analysis: a tutorial. Limnology \& Oceanography Methods, 2008, 6(11) : 572-579. DOI: 10.4319/lom.2008.6.572.

[ 18] Xu H, Jiang H. Uv-induced photochemical heterogeneity of dissolved and attached organic matter associated with cyanobacterial blooms in a eutrophic freshwater lake. Water Research, 2013, 47 (17) : 6506-6515. DOI: 10.1016/j.watres.2013. 08.021.

[19] Chen XS, Jiang T, Lu S et al. Three-dimensional fluorescence spectral characteristics of different molecular weight fractionations of dissolved organic matter in the water-level fluctuation zones of Three Gorges Reservoir Areas. Environmental Science, 2016, 37(3) : 884-892. DOI : 10.13227/j.hjkx.2016.03.012. [陈雪霜, 江蹈, 卢松等. 三峡库区消落带水体 DOM 不同分子量组分三维苂光特征. 环境科学, 2016, 37(3) : 884-892.]

[20] Devilbiss SE, Zhou Z, Klump JV et al. Spatiotemporal variations in the abundance and composition of bulk and chromophoric dissolved organic matter in seasonally hypoxia-influenced Green Bay, Lake Michigan, USA. Science of the Total Environment, 2016, 565: 742-757. DOI: 10.1016/j.scitotenv.2016.05.015.

[21] Yang GS, Ma RH, Zhang L et al. Lake status major problems and protection strategy in China. J Lake Sci, 2010, 22(6) : 799-810. DOI: 10.18307/2010.0601. [杨桂山, 马荣华, 张路等. 中国湖泊现状及面临的重大问题与保护策略. 湖 泊科学, 2010, 22(6): 799-810.]

[22] Chen XL, Zhang Y, Zhang L et al. Distribution characteristic of nitrogen and phosphorus in Lake Poyang during high water period. J Lake Sci, 2013, 25(5) : 643-648. DOI: 10.18307/2013.0504. [ 陈晓玲, 张媛, 张琍等. 丰水期鄱阳湖水体 中氮、磷含量分布特征. 湖泊科学, 2013, 25(5): 643-648.]

[23] Wang ML, Zhou WB, Hu CH et al. Status of nitrogen and phosphorus in waters of Lake Poyang Basin. J Lake Sci, 2008, 20(3) : 334-338. DOI: 10.18307/2008.0311. [王毛兰, 周文斌, 胡春华. 鄱阳湖区水体氮、磷污染状况分析. 湖泊 科学, 2008, 20(3) : 334-338.]

[24] Xiao YD, Peng WW, An Q et al. Levels of hydroxyl radicals and its purification on antibiotics in surface waters of Lake Poyang during wet and dry seasons. Acta Scientiae Circumstantiae, 2018, 38(8) : 2965-2973. DOI: 10.13671/j.hjkxxb. 2018.0166. [肖远东, 彭维维, 安琦等. 丰枯水期鄱阳湖表层水中羟基自由基浓度及其抗生素净化作用研究. 环境 科学学报, 2018, 38(8): 2965-2973.]

[25] Coble PG. Characterization of marine and terrestrial dom in seawater using excitation-emission matrix spectroscopy. Marine Chemistry, 1996, 51(4) : 325-346. DOI: 10.1016/0304-4203(95)00062-3.

[26] Cai WL, Xu XY, Du X et al. Parallel factor analysis with EEM on dissolved organic matter in chongqing section of Jialing River. Research of Environmental Sciences, 2012, 25(3): 276-281. DOI: 10.13198/j.res.2012.03.35.caiwl.009. [蔡文 良, 许晓毅, 杜涃等. 嘉陵江重庆段 DOM 三维荧光光谱的平行因子分析. 环境科学研究, 2012, 25(3) : 276-281.]

[27] Wunsch UJ, Bro R, Stedmon CA et al. Emerging patterns in the global distribution of dissolved organic matter fluorescence. Analytical Methods, 2019, 11(7) : 888-893. DOI: 10.1039/c8ay02422g.

[28] Ishii SKL, Boyer TH. Behavior of reoccurring parafac components in fluorescent dissolved organic matter in natural and engineered systems: a critical review. Environ Sci Technol, 2012, 46(4) : 2006-2017. DOI: 10.1021/es2043504.

[29] Louie SM, Spielman-sun ER, Small MJ et al. Correlation of the physicochemical properties of natural organic matter samples from different sources to their effects on gold nanoparticle aggregation in monovalent electrolyte. Environmental Science \& Technology, 2015, 49(4) : 2188-2198. DOI: 10.1021/es505003d.

[30] Mcknight DM, Boyer EW, Westerhoff PK et al. Spectrofluorometric characterization of dissolved organic matter for indica- 
tion of precursor organic material and aromaticity. Limnology and Oceanography, 2001, 46(1) : 38-48. DOI: 10.4319/lo. 2001.46.1.0038.

[31] Yan C, Liu H, Sheng Y et al. Fluorescence characterization of fractionated dissolved organic matter in the five tributaries of Lake Poyang, China. Science of the Total Environment, 2018, 637/638: 1311-1320. DOI: 10.1016/j. scitotenv. 2018. 05.099.

[32] Xu H, Zou L, Guan D et al. Molecular weight-dependent spectral and metal binding properties of sediment dissolved organic matter from different origins. Science of the Total Environment, 2019, 665: 828-835. DOI: 10.1016/j. scitotenv. 2019.02.186.

[33] Xu H, Guo L. Molecular size-dependent abundance and composition of dissolved organic matter in river, lake and sea waters. Water Research, 2017, 117: 115-126. DOI: 10.1016/j.watres.2017.04.006.

[34] Shen Z, Gong X, Jiang L et al. Analysis of the composition and source of DOM in Lake Poyang by using regional integration method. Environmental Science \& Technology, 2019, 42(5): 196-203. [申钊颖, 弓晓峰, 江良等. 利用苂光区域 积分法解析鄱阳湖 DOM 组成及来源. 环境科学与技术, 2019, 42(5) : 196-203.]

[35] Yamashita Y, Jaffé R. Characterizing the interactions between trace metals and dissolved organic matter using excitation-emission matrix and parallel factor analysis. Environmental Science \& Technology, 2008, 42(19) : 7349-7374. DOI: 10. $1021 /$ es801357h.

[36] Bai LS. Effect of dissolved organic matter on the migration and transformation of $\mathrm{Pb}$ and $\mathrm{As}$ in Nansi Lake[Dissertation]. Xuzhou: China University of Mining and Technology, 2015. [柏林森. 南四湖溶解性有机质对 Pb 和 As 迁移转化的影 响 [学位论文]. 徐州: 中国矿业大学, 2015.]

[37] Huang WD. Contents and sources of organic colloid and its effects on heavy metals behaviors in Yangtze River Estuary [Dissertation]. Shanghai: Central China Normal University, 2013. [黄文丹. 长江河口水体有机胶体含量、来源及其对 重金属行为影响的研究 [学位论文]. 上海: 华东师范大学, 2013.]

[38] Ross JM, Sherrell RM. The role of colloids in tracemetal transport and adsorption behavior in New Jersey Pinelands streams. Limnology and Oceanography, 1999, 44(4) : 1019-1034. DOI: 10.4319/lo.1999.44.4.1019.

[39] Martin J, Dai M, Cauwet G. Significance of colloids in the biogeochemical cycling of organic-carbon and trace-metals in the Venice Lagoon (Italy). Limnology and Oceanography, 1995, 40(1) : 119-131. DOI: 10.2307/2838254.

[40] Wells ML, Smith GJ, Bruland K. The distribution of colloidal and particulate bioactive metals in Narragansett Bay, Ri. Marine Chemistry, 2000, 71(1) : 143-163. DOI: 10.1016/S0304-4203(00)00046-3/.

[41] Whitehouse B, Yeats P, Strain P. Cross-flow filtration of colloids from aquatic environments. Limnology and Oceanography, 1990, 35(6) : 1368-1375. DOI: 10.2307/2837446.

[42] Zhang ZP. Study on colloidal trace metals in Taihu Lake and its influencing factors[Dissertation]. Hangzhou: Zhejiang University, 2006. [张战平. 太湖水体中的胶体态痕量金属及其影响因素研究 [学位论文]. 杭州: 浙江大学, 2006.]

[43] Dai M, Martin J, Cauwet G. The significant role of colloids in the transport and transformation of organic-carbon and associated trace-metals ( Cd, Cu and Ni) in the Rhone Delta (France). Marine Chemistry, 1995, 51(2) : 159-175. DOI: 10. 1016/0304-4203( 95 ) 00051-R.

[44] Yu X, Zhang J, Kong F et al. Identification of source apportionment and its spatial variability of dissolved organic matter in Dagu River-jiaozhou Bay Estuary based on the isotope and fluorescence spectroscopy analysis. Ecological Indicators, 2019, 102 : 528-537. DOI: 10.1016/j.ecolind.2019.03.004.

[ 45] He W, Lee J, Hur J. Anthropogenic signature of sediment organic matter probed by Uv-visible and fluorescence spectroscopy and the association with heavy metal enrichment. Chemosphere, 2016, 150: 184-193. DOI: 10.1016/j. chemosphere. 2016.01.116.

[46] Shi TR, Zhang Y, Yu T et al. Distribution of different molecular weight fractions of dissolved organic matters and their complexation with $\mathrm{Cu}$ and $\mathrm{Pb}$ in the sediment from Dianchi Lake, China. Research of Environmental Sciences, 2013,26 (2) : 137-144. DOI: 10.13198/j.res.2013.02.26.shitr.007. [石陶然, 张远, 于涛等. 滇池沉积物不同分子量溶解性 有机质分布及其与 $\mathrm{Cu}$ 和 $\mathrm{Pb}$ 的相互作用. 环境科学研究, 2013, 26(2) : 137-144.] 